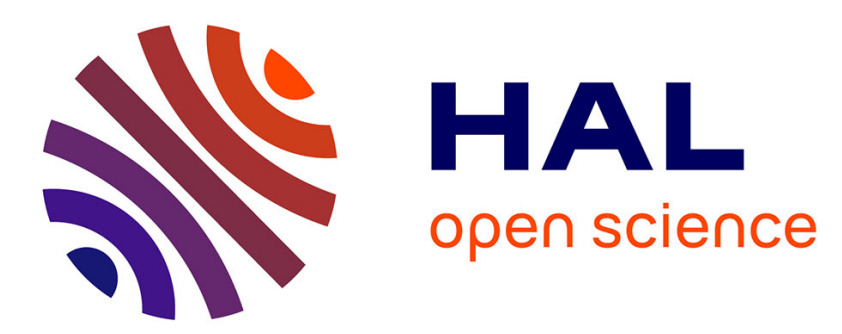

\title{
Micromechanics of wing crack propagation for different flaw properties
}

\author{
Jérôme Duriez, L. Scholtes, F.-V. Donzé
}

\section{To cite this version:}

Jérôme Duriez, L. Scholtes, F.-V. Donzé. Micromechanics of wing crack propagation for different flaw properties. Engineering Fracture Mechanics, 2016, 153, pp.378 - 398. 10.1016/j.engfracmech.2015.12.034 . hal-01865371

\section{HAL Id: hal-01865371 \\ https://hal.science/hal-01865371}

Submitted on 31 Aug 2018

HAL is a multi-disciplinary open access archive for the deposit and dissemination of scientific research documents, whether they are published or not. The documents may come from teaching and research institutions in France or abroad, or from public or private research centers.
L'archive ouverte pluridisciplinaire HAL, est destinée au dépôt et à la diffusion de documents scientifiques de niveau recherche, publiés ou non, émanant des établissements d'enseignement et de recherche français ou étrangers, des laboratoires publics ou privés. 


\section{Micromechanics of wing crack propagation for different flaw properties

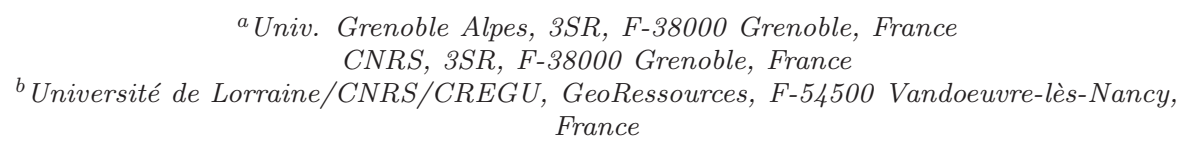

\section{Abstract}

The Discrete Element Method is used to study crack propagation in intact rock from pre-existing flaws of different natures. Damage mechanisms occurring during open and closed cracks propagation are analyzed at the local scale using an innovative micromechanical investigation. Different micromechanisms are captured, due to the development of either tensile or deviatoric states of stress in the vicinity of the flaw, which are shown to be dependent on the flaw properties. In turn, crack propagation patterns, as strength, are greatly affected by the mechanical and geometrical characteristics of the initial flaw.

9 Keywords: Rock, Discrete Element Method, Mixed mode fracture

\section{Introduction}

Rock failure occurs after little plastic deformation under unconfined conditions. Such brittle failure involves catastrophic crack propagation that results from stress concentration around flaws of different natures. These flaws may result from rock genesis, e.g. joints between rock minerals, or loading history, e.g. cracks. Among the numerous possible configurations leading to fracture generation and growth, the focus is set here on a classical configuration where a rock sample is submitted to an unconfined compressive loading in presence of a unique flaw (see Fig. 1). This corresponds to mode I+II loading, and pioneering experiments based on this configuration were undertaken mainly on

\footnotetext{
* Corresponding author

${ }^{1}$ Present address: Dept. of Civil Engineering, Univ. of Calgary, 2500 University Dr NW,

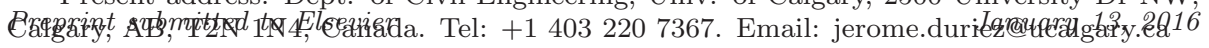



secondary cracks.

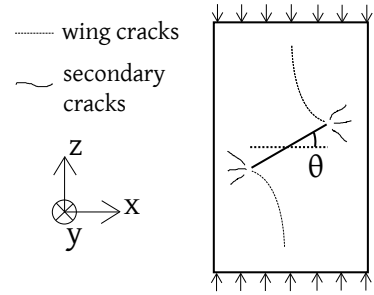

Figure 1: Compression of a pre-cracked sample.

model materials (gypsum, PMMA, glass, etc...) as presented in $[1,2,3]$. These authors observed what is now classicaly denoted as wing or primary cracks and

Wing cracks are localized crack patterns propagating along the most compressive stress direction from the flaw tips. Secondary cracks are located near the flaw tips, forming after the wing cracks and extending in a more restrained and diffuse manner compared to the latters (see e.g. $[2,4]$ ). Generally, a tensile nature is associated to wing cracks, whereas secondary cracks, sometimes denoted as shear cracks, would arise from a shear mechanism [5, 6, 4]. However, some authors may state that these secondary cracks appear through the coalescence of local tensile cracks oriented along a different direction than the wing cracks $[7,8]$.

Wing and secondary cracks have been observed with distinct shapes in model materials $[1,3,9,4]$, fragile polymers $[10,11,12]$ or marble [8]. Their occurrence might be less remarkable in other rock types such as e.g. granite [12]. Nonetheless, wing and secondary cracks are now commonly used by geomechanicians to describe crack propagation and coalescence in rocks [5, 8, 13].

Because of their consequence on the overall behavior of rock and other brittle materials, numerous models have been proposed to study crack propagation. Analytical derivations have been led, generally at the cost of elasticity hypothesis $[14,15,16]$. More complex mechanical behaviors can be handled more easily using continuous numerical modellings, such as in [17]. Nevertheless, propagating cracks are difficult to describe with continuous numerical modellings; though this can still be done using meshless methods such as XFEM [18]. 
On the other hand, discrete multi-scale models describe efficiently by nature both crack propagation and complex mechanical behavior. The inherent discrete structure of rock involving a cohesive assembly of minerals as in granite, or grains as in sandstone, is one reason to use such discrete models. Furthermore, the Discrete Element Method for instance (DEM, [19]) has proven to be an efficient modelling approach for crack propagation analysis in brittle materials $[20,21,22,23,24]$, including rock [25]. For this reason, many recent works rely on the DEM to study damage in rock, in order to reproduce experimental results such as accoustic emissions [26, 27] or constitutive behavior [28, 29]. Crack propagation from an open flaw has been studied with DEM, mainly in 2D [30, 12, 31]. In the regular lattice model of [30], wing cracks could be generated, with a limited kink. The damage patterns obtained in $[12,31]$ were less marked: this may arise from the heterogeneous strength parameters in these models, which might be related to the differences obtained experimentally for different materials.

One can note that less studies consider crack propagation from closed flaws. Experimentally, it is difficult to generate closed flaws with controlled properties [9], but some results suggest similar crack propagation patterns from open or closed flaws $[9,6,4]$. Closed flaws were simulated in DEM in 2D [32] and in 3D [33], with, however, contradictory conclusions regarding the numerical requirements for wing crack simulations. This will be discussed in section 5.2, considering different approaches to model closed flaws. As it will be emphasized in the paper, modelling closed flaws with DEM may be biaised due to the spherical shape of the discrete particles if the formulation is not upgraded.

Aiming to study crack propagation in rock with various flaw properties, our objective is twofold. First, we aim to propose an approach that is valid for either open or closed planar flaws. Second, we seek to get micro-mechanical insights on the damage mechanisms associated to wing and secondary cracks.

First, the DEM model used to simulate the rock matrix is presented in sec- 
tion 2. The model relies on previous developments [29], and its limitations are discussed. The micro-mechanical tools are also introduced. Section 3 discusses how closed flaws are simulated in the DEM model. In section 4, crack propagation is studied considering the case of open flaws, comparing the numerical results with available experimental ones. Section 5 presents mechanically consistent simulations of crack propagation from closed flaws with different mechanical properties. Finally, section 6 provides micro-mechanical insights into wing and shear cracks propagation.

All simulations are performed with the open-source code Yade [34, 35], and the geomechanics sign convention is used throughout the whole paper, considering compressive stresses and strains as positive.

\section{Rock matrix modeling}

\subsection{Model formulation}

Rock matrix is simulated using a packing of bonded spherical discrete elements (also denoted particles in the present article). The core of the model, previously presented in [29], is to some extent similar to other DEM models for rock $[25,28]$. A major difference relies in the consideration, here, of near neighbour interactions through a controlled interaction range. Indeed, interparticle bonds are created between each pair of particles $A$ and $B$ for which equation (1) is fullfilled:

$$
D_{A B}^{0} \leqslant \gamma_{\text {int }}\left(R_{A}+R_{B}\right)
$$

In equation (1), $R_{A}$ and $R_{B}$ are the radii of the two particles, $D_{A B}^{0}$ the initial distance between the two centroïds of $A$ and $B$, and $\gamma_{\mathrm{int}} \geqslant 1$ a parameter of the model. With such controlled near neighbour interactions, first proposed in [36], the average number of bonds per particle, $N$, can be predefined. This feature is motivated by the inadequate UCS/UTS ratios provided by classical DEM using spherical particles [25, 37], UCS (resp. UTS) being the uniaxial compressive (resp. tensile) strength. The mean contact number $N$ being related

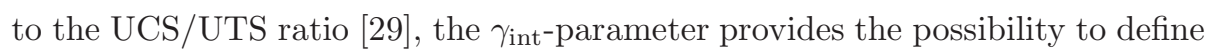
precisely $N$ and to simulate various rock types. 
As an alternative, the use of clumps (rigid aggregates) of spheres has been proposed as a solution for 2D simulations [38], but the same method might lead to less significant improvements in 3D [39]. Other solutions would be to adapt the contact laws of the parallel bond model of [25], introducing up to 10 parameters [39], or using the so-called flat joint contact model which has been developped up to now for the 2D case [40]. The use of a controlled interaction range with one scalar parameter $\gamma_{\text {int }}$ provides an efficient approach simple to formulate.

The behavior of the medium is defined through normal and tangential interaction forces acting between interacting particles. Within DEM, interaction forces are classically computed from the relative displacement between particles. $D_{A B}$ being the current value of the distance between the two centroïds, the normal force $F_{n}$ is computed from the normal relative displacement $u_{n}=D_{A B}^{0}-D_{A B}\left(u_{n}\right.$ increases when spheres get closer to each other). Both repulsive (compressive), and cohesive (tensile), normal forces are considered. In tension, normal forces can develop up to a threshold $F_{n}^{\max }$ such that:

$$
F_{n}=k_{n} u_{n} \text { while } k_{n} u_{n}>-F_{n}^{\max } ; F_{n}=0 \text { otherwise }
$$

with $F_{n}^{\max }=t A_{\text {int }}>0$ computed from the tensile strength $t$ (in $\mathrm{Pa}$ ) and $A_{\text {int }}=\pi \min \left(R_{A}, R_{B}\right)^{2}$, a surface related to the interacting particles. The normal stiffness $k_{n}$ is computed as a function of the particles radii and $Y$, a parameter of the model expressed in $\mathrm{Pa}$ :

$$
k_{n}=\frac{2 Y R_{A} R_{B}}{R_{A}+R_{B}}
$$

Equation (3) expresses the normal stiffness as the one of two spring series with stiffnesses $Y 2 R_{A / B}$, that can be interpreted as the stiffnesses of two elastic particles. In the end, $\mathrm{Y}$ is related, though different, with the bulk modulus of the numerical sample.

The tangential local stiffness $k_{t}$ is deduced from the second elastic parameter of the model, $P$ (dimensionless) such as:

$$
k_{t}=P k_{n}
$$



of our qualitative analysis.

\begin{tabular}{|c|c|c|c|c|c|}
\hline$N$ & $Y(\mathrm{GPa})$ & $P$ & $\varphi\left(^{\circ}\right)$ & $c(\mathrm{MPa})$ & $t(\mathrm{MPa})$ \\
\hline 12 & 50 & $1 / 3$ & 18 & 45 & 4.5 \\
\hline
\end{tabular}

Table 1: Considered model parameters for the intact rock.

151

The tangential force $\vec{F}_{t}$ is linearly incremented using $k_{t}$ and the incremental relative tangential displacement $\Delta \vec{u}_{t}$. Tangential forces can increase in norm up to a cohesive-frictionnal threshold $F_{t}^{\max }$ computed from the local friction angle $\varphi$ and the cohesion parameter $c$ (in Pa): $F_{t}^{\max }=c A_{\text {int }}+F_{n} \tan (\varphi)$.

Interparticle bonds may fail through tension or shear when the normal force or the tangential force reach respectively $-F_{n}^{\max }$ or $F_{t}^{\max }$. Then, the interaction disappears in the tensile regime if $F_{n}<0$, or keeps going in a compressive regime if $F_{n} \geqslant 0$. For the latter case, the behavior becomes purely frictionnal: $t$ and $c$ are set to zero. From this point, $F_{n} \geqslant 0\left(F_{n}^{\max }=0\right)$ and $F_{t}^{\max }=F_{n} \tan (\varphi)$. The same purely frictional behavior rules the interactions appearing during the simulation when spheres come in strict geometrical contact (i.e. $\gamma_{\mathrm{int}}=1$ ).

An explicit time-domain integration scheme is used to solve the equations of motion. The discrete elements are thus translated and rotated according to the interaction forces and their resulting torques using Newton's second law. Because of the dynamic formulation of the method, damping is used in the model to dissipate kinetic energy, as described in the following section.

Table 1 presents the retained parameter values. The resulting UCS, UTS and Young's modulus are respectively $70 \mathrm{GPa}, 6 \mathrm{GPa}$ and $55 \mathrm{GPa}$ (see next sections), which corresponds to a Carboniferous Limestone [41]. A detailed presentation of the calibration process can be found in [33, 29]. Note however that a complete quantitative description of this specific rock is out of the scope

\subsection{Numerical damping}

A local non-viscous damping [42] is used, introducing a damping force $\vec{F}^{d}$ in Newton's second law such that:

$$
\vec{F}^{d}=-\alpha \operatorname{sign}\left(\Sigma \vec{F}^{(t)} \cdot\left(\vec{v}^{(t)}+\frac{d t}{2} \vec{a}^{(t)}\right)\right) \Sigma \vec{F}^{(t)}
$$


$\vec{F}^{d}$ depends on the damping parameter $\alpha \in[0 ; 1], \alpha=0$ corresponding to an undamped system. This damping method facilitates quasi-static simulations, by dissipating kinetic energy in the model. Note that energy dissipation is also included in the model through sliding and brittle failure processes. Few authors present damping as an indirect modelling of other physical energy dissipation sources $[26,25]$. In this case, $\alpha$ should be considered as a model parameter that would require experimental measurements such as seismic quality factor for calibration. However, retained $\alpha$ values are in the end not consistent with this approach (see e.g. [25]).

Here, damping is considered only as a convenient numerical treatment that reduces computational costs, since it allows quasi-static conditions with higher loading rates. Then, its possible influence on the results is assessed by simulating uniaxial compression tests for different values of $\alpha$, with the parameters of Table 1.

Uniaxial tests simulations consist in the loading of a parallellepipedic sample of spherical particles between two rigid platens (see the Fig. 3). Deformation of the sample is caused by the movement of one platen toward the other at a constant speed. The platens are frictionless to favour a homogeneous deformation inside the sample. Details about the simulation procedure and the influence of the platen friction angle were given in [29]. Here, stresses deduced from the force acting on the moving platen, and the one deduced from Love-Weber homogeneization formula $[43,44]$ are equal, confirming an acceptable level of the homogeneity and quasi-staticity within the model.

As shown in Fig. 2, the pre-peak behavior is not affected by damping, but the UCS increases (up to $30 \%$ here) according to $\alpha$. Failure occurs along with a brutal increase in the number of broken bonds (Fig. 2). At the stress peak, the perturbations induced by the rupture of one interaction trigger a chain reaction leading to the failure of a set of bonds that will consequently bisect the sample [26]. Such chain reaction is inhibited by the damping, thus delaying failure and increasing the stress that may be reached (Fig. 2). Similar trends were reported in $[26,45,28]$. Note that different simulations run with different loading rates 


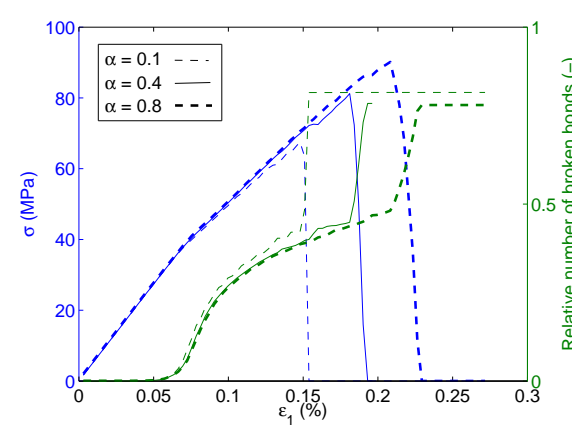
peak in every case.

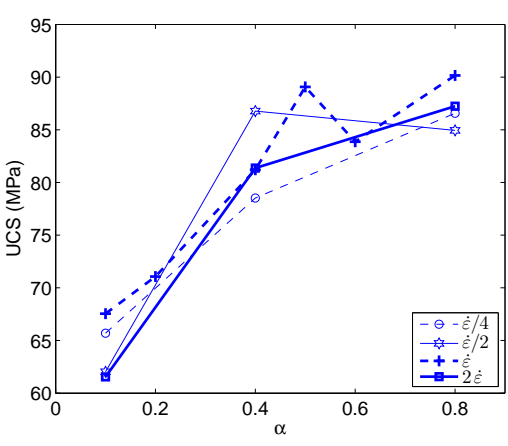

(a) Reference loading rate case. The number (b) UCS for different loading rates around the of broken bonds is relative to the initial num- reference $\dot{\varepsilon} \approx 7.4 \times 10^{-4} s^{-1}$ ber of bonds

Figure 2: Damping influence for uniaxial compression test simulation.

show the same trends, which means that quasi-staticity is ensured up to the

The significant UCS variations reveal an inherent problem of the model formulation, due to the introduced damping and the brittleness of the contact law; even though such model is quite classical for rock simulations $[26,25,45$, 28, 29]. As such, a constant low-level damping is used throughout this study: $\alpha=0.2$, which is rather limited compared to other works: $\alpha=0.7$ in [25], $\alpha=0.4$ in [29]. Doing so, we approach the behavior of an undamped model, keeping reasonnable computational costs.

\subsection{Discrete element size influence}

As, e.g. for computational cost reasons, the particles used in the simulations do not correspond in number and in size to real physical entities, the influence of particle size on the results has to be considered. Uniaxial compressive tests were performed on three different numerical samples (Table 2) using the parameters presented in Table 1.

Samples 1 and 2 contain around 21000 particles with a mean diameter $D \approx$ $22 \mathrm{~cm}^{\star}$. However, they do not involve the same packing due to the random

\footnotetext{
${ }^{\star}$ Length units are given for consistency, but they do not matter and absolute dimensions
} 


\begin{tabular}{|c|c|c|} 
Designation & Number of elements & Mean diameter $(\mathrm{m})^{\star}$ \\
\hline Sample 1 & 20689 & 0.215 \\
Sample 2 & 20689 & 0.215 \\
Sample 3 & 124137 & 0.118
\end{tabular}

Table 2: Samples used for studying size dependency. For all samples, $L_{x} \approx 5.4 \mathrm{~m}, L_{y} \approx 2.4 \mathrm{~m}$, $L_{z} \approx 10.8 \mathrm{~m}$, and a uniform distribution of radii is used with $D_{\max } / D_{\min } \approx 1.86$. The $(x, y, z)$ framework is depicted in Fig. $3, z$ being the loading direction.

the mean diameter only.

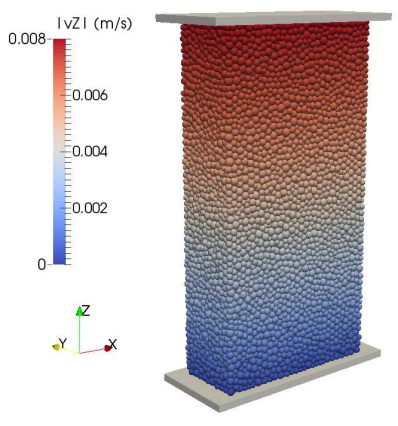

(a) Samples 1 or 2

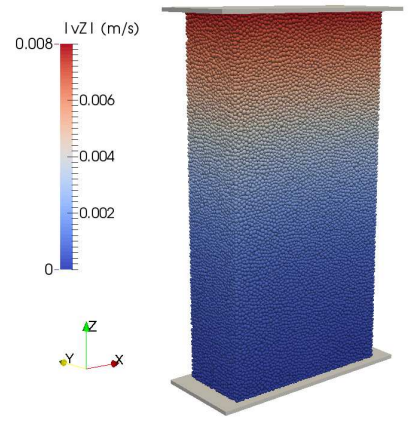

(b) Sample 3

Figure 3: Different samples loaded in uniaxial compression.

As shown in Fig. 4, the same macroscopic elastic stiffness is obtained whatever the mean diameter. This results from the introduction of the radii of the particles in equation (3). Concerning the plastic behavior, both samples with the same mean diameter $D$ exhibit a similar strength with a difference of about $5 \%$ for the stress peak. However, greater differences appear for the third sample that involves smaller particles with mean diameter $D^{\prime}<D$. The stress peak

are not intended to correspond to physical entities. 


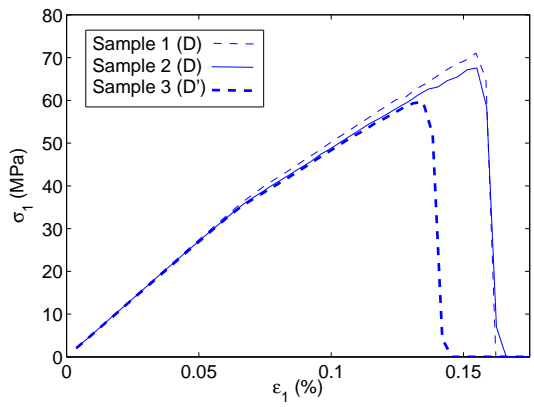

Figure 4: Uniaxial compressive test for different samples $\left(D^{\prime} \approx D / 2\right)$ with same parameters.

is here reduced by about $13 \%$ when the mean diameter decreases. This is related to the elastic-brittle interaction laws that induce an influence of the mean diameter on the fracture toughness, explained as follows.

For illustrative purposes, let us consider a monodisperse packing of bonded spheres of diameter $D$ (Fig. 5). A crack of surface $A_{c}$ exists in the packing, due to previous bond breakages (Fig. 5(a)). The rupture of another bond induces a growth of the crack surface by $d A_{c}$ (Fig. $5(\mathrm{~b})$ ). Under pure tensile loading (no shear forces), the energy released during this bond breakage is equal to $E=1 / 2\left(F_{n}^{\max }\right)^{2} / k_{n}$.

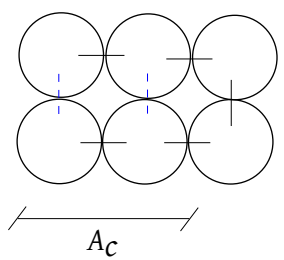

(a) Before crack propagation

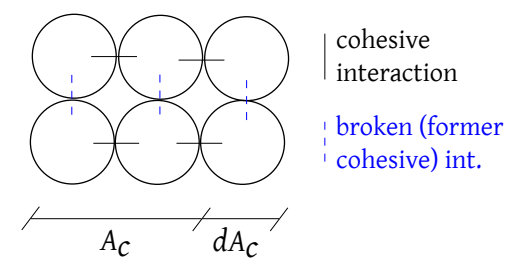

(b) After crack propagation

Figure 5: Crack propagation in a DEM model.

Within our model, the critical fracture energy [46] $\mathcal{G}_{c}=E / d A_{c} \propto E / D^{2}$ depends finally on the model parameters $t, Y$ and on the sphere diameter $D$ such that:

$$
\mathcal{G}_{c} \propto \frac{t^{2}}{Y} D
$$

227 Thus, for what concerns localized failure mechanisms, the discrete model suffers 
from a size dependency: the critical surface energy is proportional to the mean diameter $D$, or, equivalently, the fracture toughness is proportional to $\sqrt{D}$. Using a different approach, the same conclusion has been drawn in [25]. If the toughness was to be set without any particle size-dependency, the interaction law should be modified, introducing another parameter, as suggested in [21]. Nevertheless, the following sections will show that the model still has advantages to study qualitatively crack propagation. Quantitative comparisons can also be led as long as the same discretization size is kept.

\subsection{Micro-mechanical insight}

For any material system, it is possible to define a symmetric tensor that expresses the power of internal forces, that is thus related to a stress tensor [47, 48, 49]. For a system made up of one particle $p$ at equilibrium under interaction forces related to several contact points $c$, the symmetric internal moment tensor $\boldsymbol{M}^{\boldsymbol{p}}[48,49]$ can be used, expressed by:

$$
M^{p}=\sum_{c} \vec{x}^{c} \otimes \vec{f}^{c}
$$

with $\vec{x}^{c}$ the position of the interaction points (from the center of the particle $p$ ) where the interaction forces $\vec{f}^{c}$ apply. A stress tensor at the particle scale, denoted as particle stress tensor, is then derived as:

$$
\sigma^{p}=-1 / V^{p} M^{p}
$$

with $V^{p}$ the volume of the particle (the minus sign is set to obey the geomechanical sign convention). For illustration purpose, Table 3 gives two examples of $\boldsymbol{\sigma}^{p}$ values.

Considering a set $S$ of particles, the related internal moment tensor $\boldsymbol{M}^{S}$ is the sum of the internal moment tensors of each particle included in $S$. If $S$ contains enough particles so that it can be considered as a REV, a direct link appears between $M^{S}$ and the Cauchy stress tensor [49], which may justify the definition of $\boldsymbol{\sigma}^{p}$. However, one particle can not constitute a REV for a DEM assembly, then $\boldsymbol{\sigma}^{p}$ is a local value that relates to the mechanical state of the assembly in a qualitative manner only. 


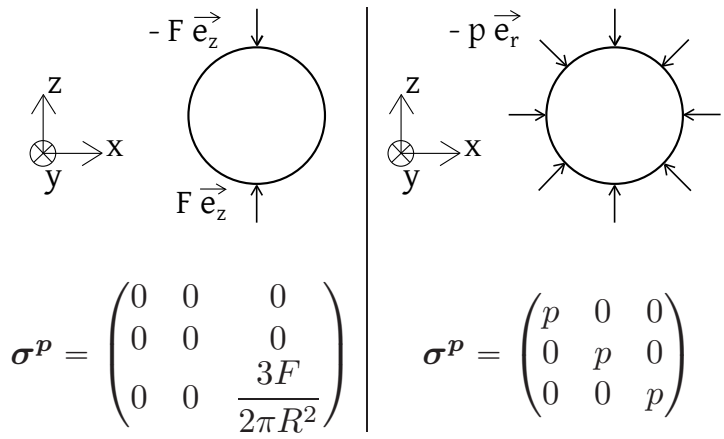

Table 3: $\boldsymbol{\sigma}^{\boldsymbol{p}}$ for two given micro-mechanical loadings: opposite forces, or uniform pressure.

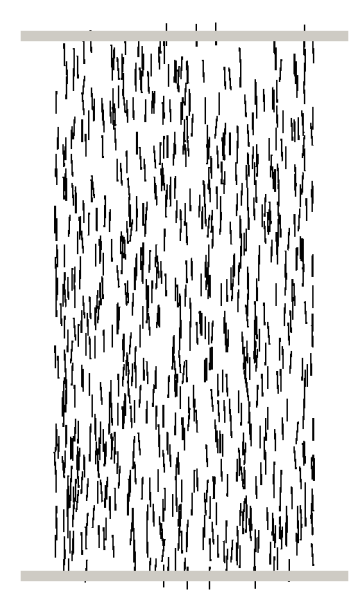

(a) Direction of major principal (b) Heterogeneity of the mastress (most compressive) jor principal stress

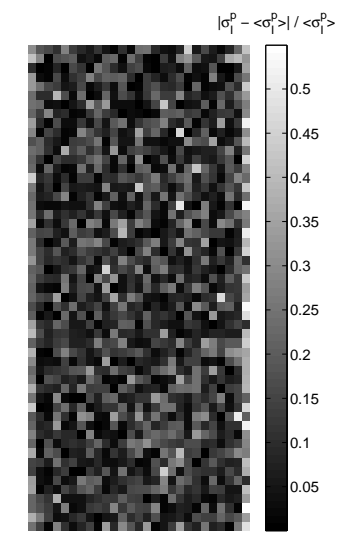

Figure 6: Micro-mechanical state of the DEM model in the pseudo-elastic phase of an uniaxial compression test.

260

261

262

The field of major (most compressive) principal stresses $\sigma_{I}^{p}$ computed during the pseudo elastic phase (pre-peak) of a uniaxial compression test is presented in Fig. 6 (details about such representations are given in Appendix A). Firstly, it can be observed that the principal directions of $\sigma_{I}^{p}$, at the particle scale, conform to the principal stress direction imposed at the macroscopic scale (Fig. 6(a)). Secondly, the major principal particle stresses are here in a certain extent

uniform, as might be expected from such homogeneous test (Fig. 6(b)). Indeed, $\sigma_{I}^{p}$ values differ from the mean $\left\langle\sigma_{I}^{p}\right\rangle$ by $50 \%$ maximum. Finally, the data 
set shows that both other principal stresses are similar and negligible compared with $\sigma_{I}^{p}$, for all particles.

These results support the use of $\boldsymbol{\sigma}^{p}$ as a qualitative micro-mechanical insight to evaluate the stress distribution at the particle scale. The method will thus be used in section 6 to analyze crack propagation.

\section{Rock discontinuities modeling}

Pre-existing discontinuity surfaces are handled in a specific manner inside the rock model. A smooth-joint model (SJM, [50]) is applied to the joint interactions that concern particles located on both sides of any discontinuity surface. Using the SJM, the normal and tangential vectors of the interaction are rotated and defined according to the surface of interest, rather than upon the contact geometry of the spherical particles. The elastic-plastic constitutive relations presented in section 2 hold for joint interactions, with adequate expressions of normal and tangential relative displacements. Namely, for such interactions $u_{n}=\left(\overrightarrow{A B}^{0}-\overrightarrow{A B}\right) \cdot \vec{n}$ with $\vec{n}$ the normal vector to the discontinuity surface, from center $A$ to center $B . \vec{n}$ is thus different from $\overrightarrow{A B} /\|\overrightarrow{A B}\|$. The plastic behavior, defined according to a friction angle $\phi$ and a dilatancy angle $\psi$, is independent of the discretization of the model [33]. In order to get rid of the size dependency also in the elastic domain, the elastic local stiffnesses $k_{n}$ and $k_{t}$ are given by:

$$
\begin{aligned}
& k_{n}=K_{n}^{j} A_{\mathrm{int}} \\
& k_{t}=K_{t}^{j} A_{\mathrm{int}}
\end{aligned}
$$

with the interface parameters $K_{n}^{j}$ and $K_{t}^{j}$ expressed in $\mathrm{Pa} / \mathrm{m}$.

Compression and shear tests were conducted on a numerical rock joint to confirm the discretization-independence. A planar joint is defined in a parallelepipedic sample by assigning a SJM to all interactions located across the corresponding surface (Fig. 7). The particles lying on each side of the surface are clumped together, in order to form two rigid blocks on each side of the rock joint. With this method, different from the ones used in $[51,33]$, the local parameters of the joint can be tested directly without any influence of the matrix 
parameters (the latter is considered as a rigid body). The joint normal and

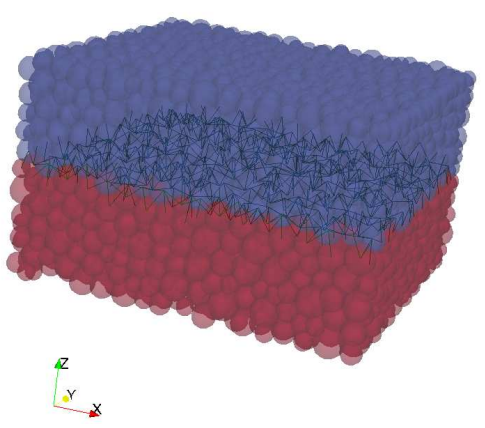

(a) Model A

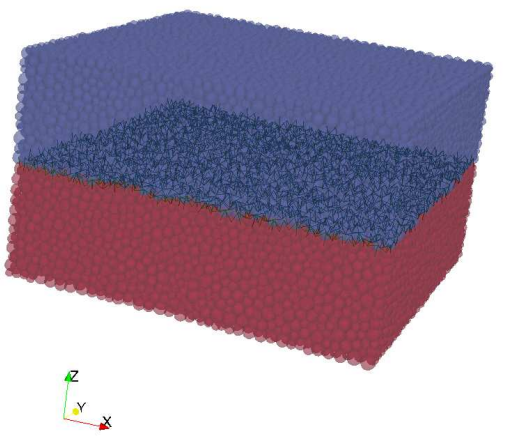

(b) Model B

Figure 7: Particles (spheres) and joint interactions (lines) of the rock joint discrete models. Model A describes the joint surface with particles of mean diameter $D \approx 0.63$. Model $\mathrm{B}$ involves particles with $D \approx 0.29$.

tangential stresses, denoted $\sigma$ and $\tau$, are directly deduced from the interacting forces occurring between the clumps. The normal and tangential relative displacements are denoted $u$ and $\gamma$ respectively.

The rock joint is first normally loaded through a compression along the $\vec{z}$ axis (Fig. 7): $d u=c s t>0 ; d \gamma=0$. The compression is performed at constant speed up to $\sigma \approx 1 \mathrm{MPa}$. A second phase of constant normal displacement (CND) shear mode $(d u=0 ; d \gamma=c s t>0)$ is then applied along the $\vec{x}$ axis, Fig. 7. The constitutive behavior of the model is presented in Fig. 8. It corresponds to an elastic-plastic rock joint with two elastic stiffnesses $\left(K_{n}^{g l o b} ; K_{t}^{g l o b}\right)$ and a plastic behavior with constant yield surface $\tau=\sigma \tan (\phi)$ and flow rule $d u^{p} /\left|d \gamma^{p}\right|=$ $-\tan (\psi)$. The plastic behavior is obtained during shear displacement from $(\gamma, \tau) \approx(9 \mathrm{~mm}, 0.7 \mathrm{MPa})$. Appendix B derives the equations of the curves that are plotted along the model's response in Fig. 8.

As shown in Fig. 8(a) and 8(b), the macroscopic elastic stiffnesses $K_{n}^{g l o b}$ and $K_{t}^{g l o b}$ can be considered to be unaffected by the mean diameter of the numerical sample. They depend on the micro-parameters $K_{n}^{j}$ and $K_{t}^{j}$, and on the ratio between the sum of all interaction surfaces $A_{\text {int }}$ and the apparent joint surface; which is directly related to the density of the packing. This dependence on the 


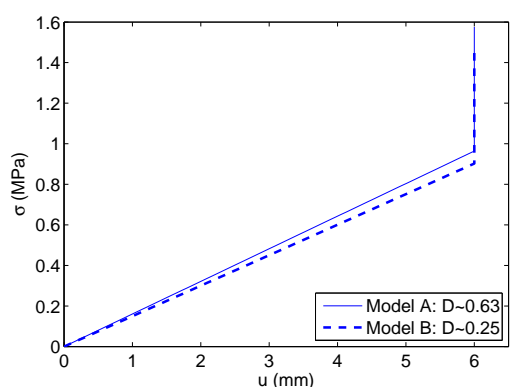

(a) $\sigma(u)$

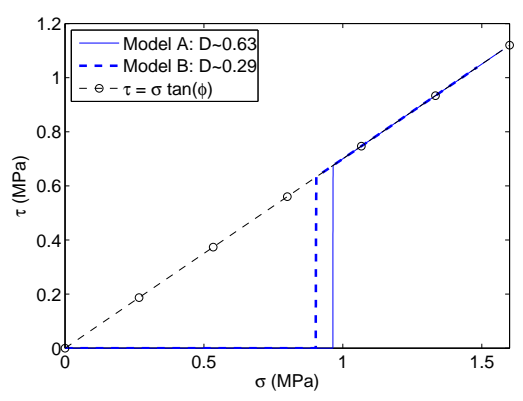

(c) $\tau(\sigma)$

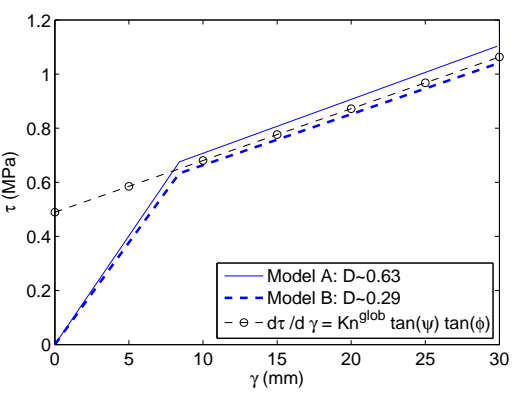

(b) $\tau(\gamma)$

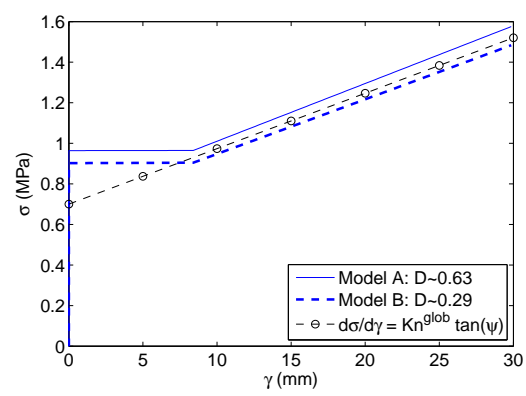

(d) $\sigma(\gamma)$

Figure 8: Behavior of two rock joint discrete models involving two different samples. All computations are made with $K_{n}^{j}=50 \mathrm{MPa} / \mathrm{m}, K_{t}^{j}=K_{n}^{j} / 2, \phi=35^{\circ}, \psi=10^{\circ}$. 
packing density induces a difference between $K_{n}^{j}$ and $K_{n}^{g l o b}$ such that: $K_{n}^{g l o b} \approx$ $155 \pm 5 \mathrm{MPa} / \mathrm{m}$ while $K_{n}^{j}=50 \mathrm{MPa} / \mathrm{m}$. However, equality between other microparameters and induced macro-properties is obtained: $K_{t}^{g l o b} / K_{n}^{g l o b}=K_{t}^{j} / K_{n}^{j}=$ 0.5, and the macro-plastic parameters $\phi$ and $\psi$ are the same as those introduced at the interaction scale. This direct correspondance is remarkable in DEM and has to be pointed out.

\section{Crack propagation from an open flaw}

DEM simulations of open pre-existing cracks is straightforward: the particles are simply removed at the flaw location. Here, the three samples presented in Table 2 are subjected to uniaxial compression, including a flaw located at their center and persistent through the $\vec{y}$ direction. The flaw length, in the $(\vec{x}, \vec{z})$ plane, is $20 \%$ of $L_{z}$.

\subsection{Strength of pre-cracked samples with open flaws}

The uniaxial compressive tests performed on samples with an open flaw show a continuous increase of the strength with the flaw orientation $\theta$ (Fig. 9(a)). Such trend is in qualitative agreement with experimental results obtained in [12] (Fig. 9(b)), and with discrete modelings performed by [12, 31]. One can note moreover that this result is not affected by the discretization of the model, i.e. the numerical assembly.

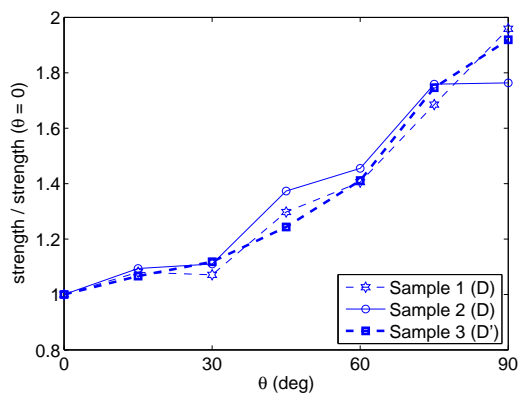

(a) DEM model for different samples

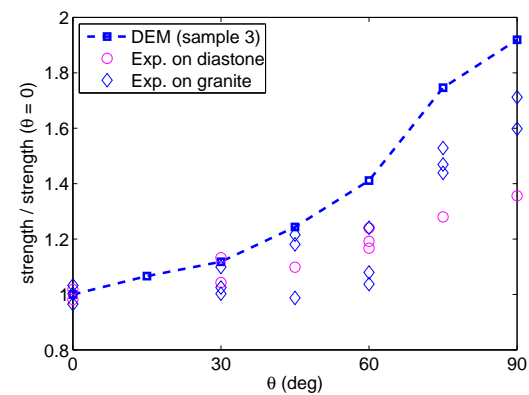

(b) DEM vs experiments (exp. data by [12])

Figure 9: Strength of pre-cracked samples (open cracks) with different crack inclinations. 
Quantitatively, different values of the $U C S\left(\theta=90^{\circ}\right) / U C S\left(0^{\circ}\right)$ ratio may be obtained: around 2 in the present numerical study, 1.2 in [31] and 1.3 to 1.7 in [12]. This difference may be related to the geometry of the open flaw that is different in every case.

\subsection{Crack propagation patterns from open flaws}

For flaw inclinations less than $60^{\circ}$, distinct wing and secondary cracks can be observed (Fig. 10), resulting from the coalescence of local bond breakages (hereafter denoted as microcracks). This result is in accordance with previous experimental works $[2,4,8,13]$. The location of the wing crack initiation depends on the inclination of the pre-existing flaw towards the direction of the major principal stress. For $\theta=0^{\circ}$, the crack propagates from the middle of the flaw. For increasing $\theta$-values, the initiation of the wing crack moves along the flaw to finally reach the flaw tip as observed experimentally [8, 4].

For higher inclinations $\left(\theta \geqslant 60^{\circ}\right.$ here), damage first initiates with a localized pattern, at the flaw tips. Then, secondary cracks appear nearby and prevent localization, so that an unique diffuse pattern develops afterwards (Fig. 10).

In our DEM simulations, all microcracks correspond to local tensile bond failures. This is directly related to the interparticle bond strength properties which were calibrated to ensure the macroscopic behavior to be representative of a brittle rock (carboniferous limestone), with the local tensile strength that is significantly smaller than the local shear strength. Such a result would support the idea that the secondary cracks, or shear cracks, appear in fact through the coalescence of local tensile cracks oriented along another direction than the wing cracks $[7,8]$. This point will be discussed in section 6.1 .

Note that the obtained crack propagation patterns do not depend on the discrete element size (Fig. 11).

\section{Crack propagation from a closed flaw}

Closed flaws can be simulated in DEM by removing the cohesive feature of all interactions located along the flaw surface as proposed by [52, 25, 32]. For 

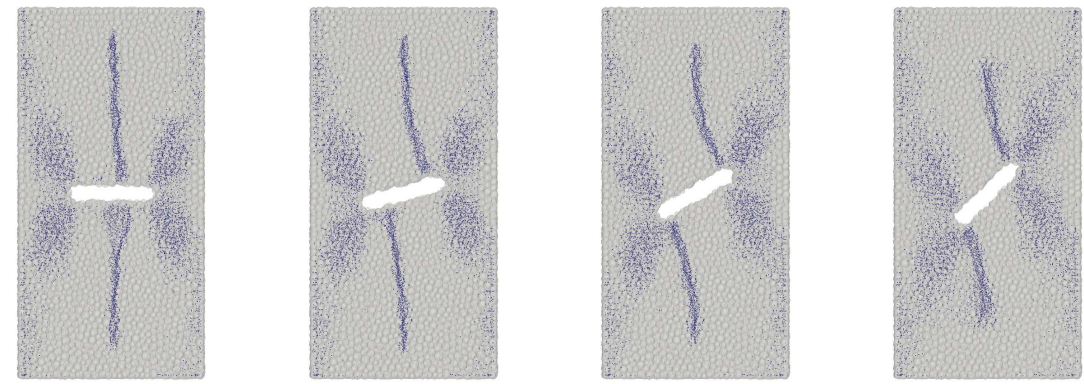

(a) $\theta=0^{\circ}(29 \mathrm{MPa})$

(b) $\theta=15^{\circ}(31 \mathrm{MPa})$

(c) $\theta=30^{\circ}(31 \mathrm{MPa})$

(d) $\theta=45^{\circ}(37 \mathrm{MPa})$
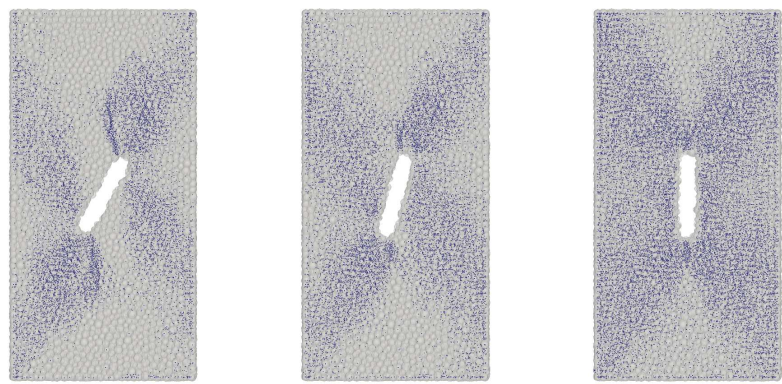

(e) $\theta=60^{\circ}(40 \mathrm{MPa})$

(f) $\theta=75^{\circ}$

$(48 \mathrm{MPa})$

(g) $\theta=90^{\circ}(56 \mathrm{MPa})$

Figure 10: Crack propagation patterns from open flaws: blue dots correspond to broken interparticle bonds locations. For $\sigma$ values all in the range [0.64 UCS; 0.71 UCS]. Strengths for each case are indicated in parenthesis in corresponding labels.
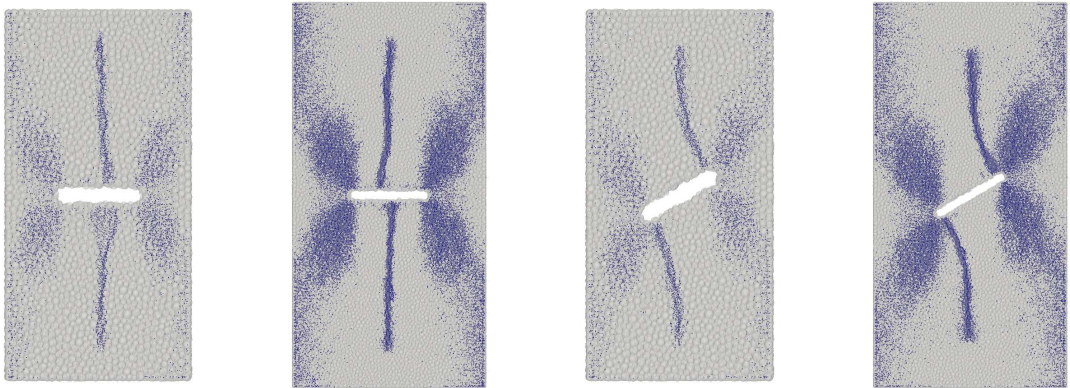

(a) $\theta=0^{\circ}$, sample 1: (b) $\theta=0^{\circ}$, sample 3: (c) $\theta=30^{\circ}$, sample 1: (d) $\theta=30^{\circ}$, sample
$\mathrm{D}(29 \mathrm{MPa})$
$\mathrm{D}^{\prime}<\mathrm{D}(25 \mathrm{MPa})$
$\mathrm{D}(31 \mathrm{MPa})$
3: $\mathrm{D}^{\prime}<\mathrm{D}(28 \mathrm{MPa})$

Figure 11: Crack propagation pattern from open flaws, obtained for different model discretizations. At $\sigma=21 \pm 1 \mathrm{MPa}$ (peak stresses of each case are indicated in label). 


\subsection{Strength of pre-cracked samples with closed flaws}

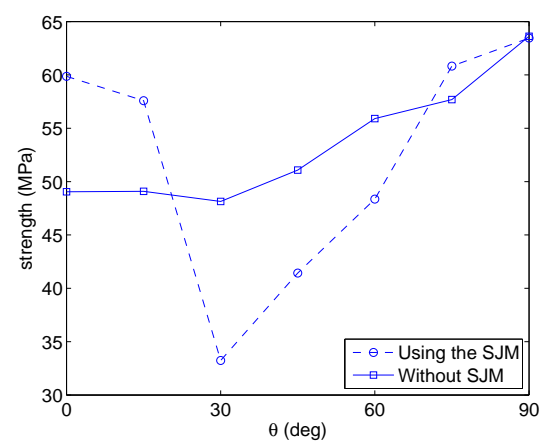

Figure 12: Strength of the pre-cracked sample, considering two closed flaw models $\left(\phi=18^{\circ}\right)$. Strength of the intact sample was $71 \mathrm{MPa}$.

such approach, due to the spherical geometry of the particles, the influence of the discretization of the model is questionable since the simulated flaw surface present a roughness that depends on the size distribution of the particles located on each side of the discontinuity surface. To discuss this point, the use of the SJM is compared with this existing approach.

Again, uniaxial compression tests are considered, using the assemblies presented in Table 2. Because the flaw is closed, its mechanical properties have to be determined. All simulations were performed here with $K_{n}^{j}=K_{t}^{j}=5$ $\mathrm{GPa} / \mathrm{m}, \psi=0^{\circ}$ and different values of $\phi$ for the interactions making up the flaw surface. Whatever the approach considered, classic contact model or smooth joint model, all simulations were performed using the same non-cohesive local properties along the flaw interactions. Using the SJM, the contact geometry of these interactions was additionally rotated according to the flaw geometry (see section 3$)$.

As expected, depending on the approach chosen for describing the closed flaw, the responses are different (Fig. 12). For low flaw inclinations $\left(\theta \leqslant 15^{\circ}\right)$, the use of the SJM leads to higher strengths, compared to the classical contact model. With the SJM, the joint interactions are all oriented perpendicular to the flaw and are thus all parallel to the direction of the loading. With- 
out the SJM, the orientations of the interactions along the flaw are randomly distributed, which leads to a lower resistance against the vertical loading. For intermediate inclinations, a drastic decrease of the strength, explained hereafter, is captured using the SJM. Without the SJM, there is a continuous, slight, increase of the strength according to $\theta$, which is in fact similar to the open flaw case (see previous Fig. 9). In addition, the results obtained without the SJM are significantly affected by the discretization. Indeed, the strength variation with the flaw inclination shows different trends depending on the numerical sample (Fig. 13).

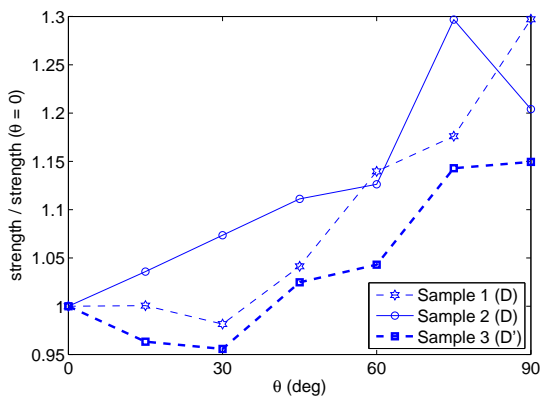

Figure 13: Strength of the pre-cracked sample for different orientations $\theta$ (see Fig. 1): closed flaw $\left(\phi=18^{\circ}\right)$ described removing cohesive interactions across the flaw, without SJM.

(3)

On the contrary, using the SJM, very few differences appear using different samples (Fig. 14(a)). Moreover, a mechanically consistent description of the flaw is clearly obtained. In every case, the strength reaches a minimum for an orientation related to the flaw friction angle (Fig. 14(b)). This results from the compressive loading, that imposes mainly vertical chains of contact forces between discrete elements (see e.g [53]). And, for $\theta>\phi$, sliding occurs for all joint interactions carrying vertical contact forces. Whereas there is no restriction for vertical contact forces along the flaw while $\theta \leqslant \phi$. For this reason, an approximatively constant strength is obtained in the inclination range $\theta \leqslant \phi$, while the strength of the sample is significantly reduced for $\theta>\phi$. This sound result can not be retrieved without the SJM, as shown in Fig. 12 and 13. 


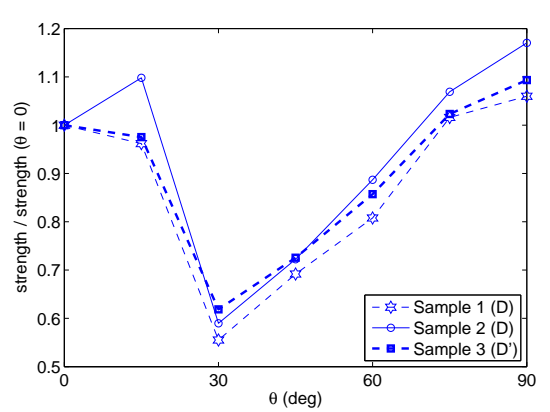

(a) For different samples $\left(\phi=18^{\circ}\right)$

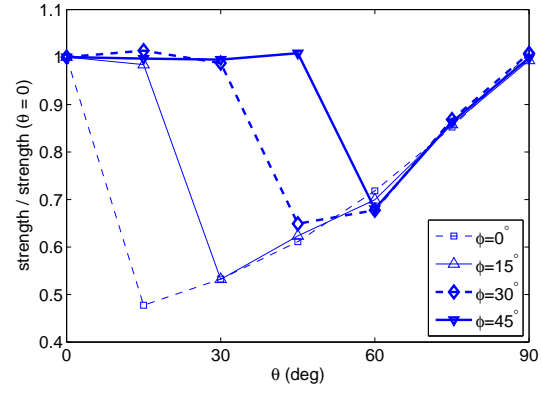

(b) For different $\phi$ values

Figure 14: Strength of the pre-cracked sample using the SJM.

\subsection{Crack propagation patterns from closed flaws}

Simulating closed flaws using the SJM, crack propagation depends on both $\theta$, the flaw inclination, and $\phi$, the flaw friction.

The fracturing patterns obtained for $\phi=0^{\circ}$ and different values of $\theta$ are shown in Fig. 15. No localized wing cracks form from a horizontal closed flaw, as opposed to the open flaw case. For other inclinations the patterns obtained with the closed flaw are in a certain extent similar to the ones obtained for an open flaw. Again, fracturing localizes through wing cracks for intermediate orientations $\left(0^{\circ}<\theta<60^{\circ}\right)$. However, the wing cracks here always initiates from the tips of the flaw and show a limited kink. Using another numerical method (the displacement discontinuity method), Shen et al. [9] showed an influence of the stiffness of the flaw surface on the curvature of the wing crack: softer flaws were shown to induce more curved wing cracks patterns, the extreme case being the case of an open flaw.

Delayed with respect to the straight localized wing cracks, zones of secondary cracks can also be observed.

For higher inclinations $\left(\theta>60^{\circ}\right.$ here), fracturing is diffuse throughout the whole sample, almost as if there was no flaw.

Using other values for the flaw friction angles $\left(\phi>0^{\circ}\right)$ demonstrates that the SJM describes wing cracks propagation for intermediate orientations if and 

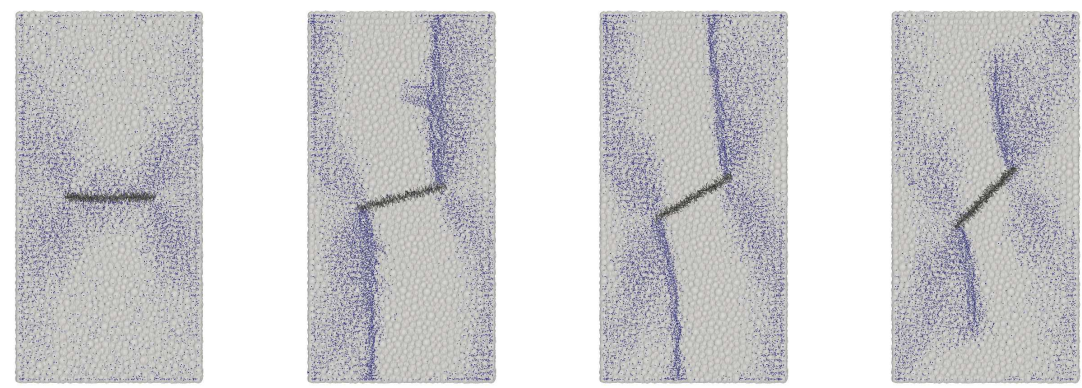

(a) $\theta=0^{\circ}(55 \mathrm{MPa})$ (b) $\theta=15^{\circ}(33 \mathrm{MPa})$ (c) $\theta=30^{\circ}(41 \mathrm{MPa})$ (d) $\theta=45^{\circ}$ (41 $\left.\mathrm{MPa}\right)$
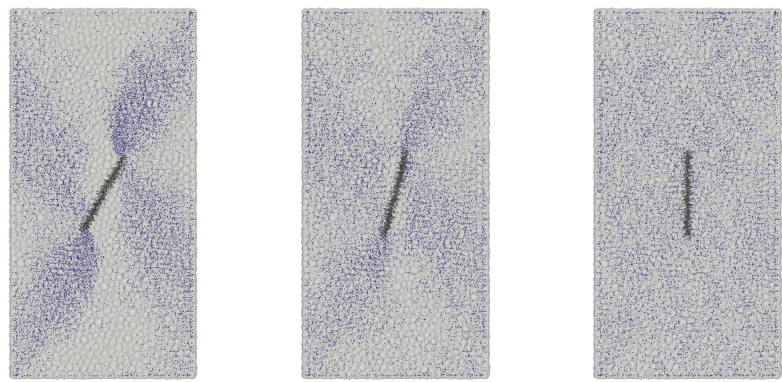

(e) $\theta=60^{\circ}(48 \mathrm{MPa})$ (f) $\theta=75^{\circ}(58 \mathrm{MPa})$ (g) $\theta=90^{\circ}(58 \mathrm{MPa})$

Figure 15: Closed crack propagation patterns at different inclinations for $\phi=0^{\circ}$, using the SJM. $\sigma$ values are all in the range [0.6 UCS; 0.7 UCS]. Strengths for each case are indicated in parenthesis with the corresponding labels. The closed flaw appears in black at the middle of the sample. 


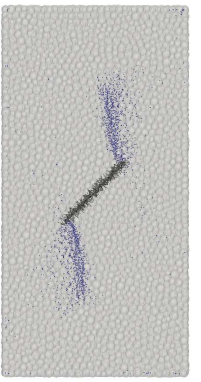

(a) $\varepsilon_{z} 0.04 \% ; \sigma_{z} \in[19 ; 20] \mathrm{MPa}$. Left: $\phi=0^{\circ}$. Right: $\phi=30^{\circ}$.

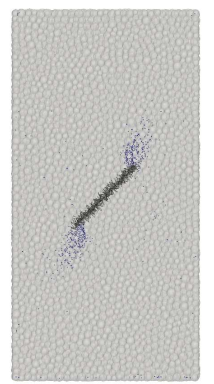

(b) $\varepsilon_{z} 0.06 \% ; \sigma_{z} \in[28 ; 29] \mathrm{MPa}$.

Left: $\phi=0^{\circ}$. Right: $\phi=30^{\circ}$.

Figure 16: Flaw friction angle influence on the propagation of a closed flaw $\left(\theta=45^{\circ}\right)$. UCS is $41 \mathrm{MPa}$ for $\phi=0^{\circ}$ and $46 \mathrm{MPa}$ for $\phi=30^{\circ}$.

429

only if sliding occurs along the flaw $\left(\phi<\theta<60^{\circ}\right)$. When sliding along the flaw is prevented because $\phi \geqslant \theta$, diffuse damage takes place throughout the whole sample and no localized crack develops, as for high inclinations and $\phi=0^{\circ}$ (Fig. 15). Such behavior is in agreement with several experiments $[9,6,4]$.

When sliding is enabled $(\phi<\theta)$, the fracturing pattern is modified by the frictional strength along the flaw. While increasing $\phi$ value, microcracks tend to propagate from the flaw tips in a unique diffuse manner. The two fracturing steps (localized wing cracks followed by diffuse secondary cracks) observed for open flaws or frictionless closed flaws can hardly be distinguished. Fig. 16 illustrates the differences observed for $\theta=45^{\circ}$ with $\phi=0$ and $30^{\circ}$.

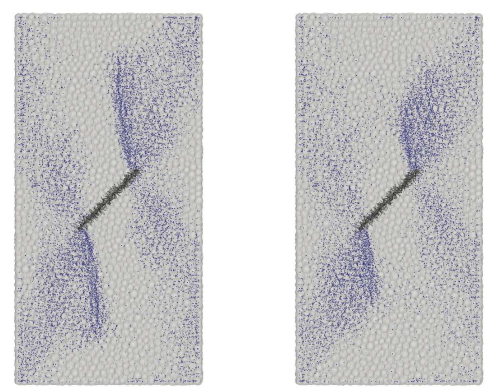

This influence of the flaw friction angle emphasizes the advantage of using the SJM for simulations of closed crack propagation using spherical discrete elements. Without the SJM, the flaw friction angle can not be precisely assigned to the model, and inadequate fracturing patterns might be obtained.

This may explain why here, as in [33], wing cracks are obtained without taking into account a transfer moment law in the DEM; contrary to what has been deduced from a previous discrete study [32] where closed flaws are simulated without the SJM, by only debonding the elements. Without any friction 

crack to develop.

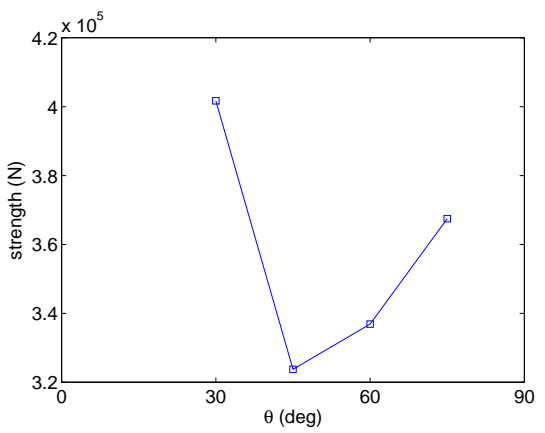

Figure 17: Strengths of pre-cracked samples with closed flaws simulated without the SJM. From data by [32].

441

defined at the contact scale along the flaw, the strength data from this previous study suggest finally a resultant flaw friction angle belonging to $\left[30 ; 45^{\circ}\right]$ (Fig. 17). According to our results, such value of the flaw friction angle prevents wing

\section{Micromechanical discussion}

The micro-mechanical quantities presented in section 2.4 are used here to give some insights into crack propagation processes. While the model includes damage through microcracking occurring at contacts, i.e. at an interparticle scale, $\boldsymbol{\sigma}^{\boldsymbol{p}}$ is defined at the particle scale, describing the mechanical state of one given particle surrounded by a set of contacts. However, these micro-mechanical fields represent a valuable meso-scale characterization of the stress state in the medium.

\subsection{Micro-mechanics of open flaw propagation}

First of all, using the micro-mechanical values, the DEM model is able to capture the stress concentration caused by the flaw, similarly to any continuous method (e.g. [54]). Let's consider, for instance, the sample with an open flaw inclined at $30^{\circ}$ (Fig. 18). The heterogeneity of the major particle stress, quantified by $\left|\sigma_{I}^{p}-<\sigma_{I}^{p}>\right| /<\sigma_{I}^{p}>$, reaches 3 here (Fig. 18(b)), while it was limited to 0.5 for a homogeneous sample (Fig. 6(b)). The presence of the pre-existing 

values, with shear and wing cracks being different in nature.

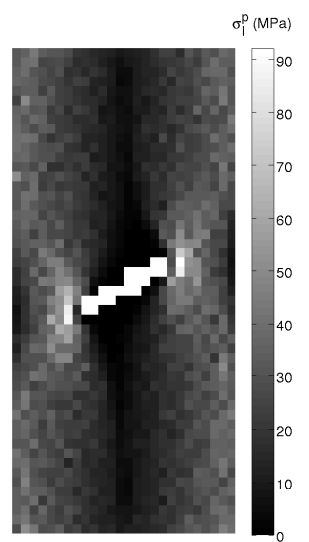

(a) Major principal stress (most compressive) map
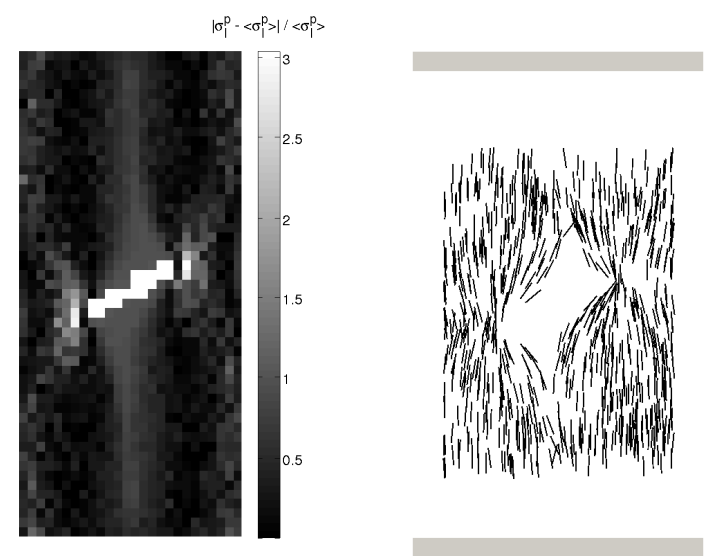

(b) Variations of the major principal stress
Directions of the major principal stress around the flaw

Figure 18: Micro-mechanical stress for a sample with an open flaw $\left(\theta=30^{\circ}\right)$ under compression $(\sigma \approx 22 \mathrm{MPa})$. See Fig. 10 for corresponding crack pattern.

460

On one hand, shear - or secondary - cracks tend to develop in highly compressed zones, i.e. zones sustaining high deviatoric stresses for such unconfined loading. Figure 19 depicts a direct comparison between the crack pattern and the particles sustaining the greatest deviatoric stresses $s^{p}$ throughout the model, emphasizing the shear nature of the secondary cracks. On the other hand, wing cracks are clearly not related to shear local stresses.

As a matter of fact, wing cracks develop where particles sustain a tensile loading. To emphasize this point, we now consider the quantity $\left(\sigma_{I I I}^{p}+\sigma_{I}^{p}\right)$ for each particle. Negative values correspond to particles with the most tensile stress $\sigma_{I I I}^{p}$ negative (i.e. corresponding actually to tension) and greater in absolute value than the most compressive stress $\sigma_{I}^{p}$. As seen in Fig. 20, there

is a very good agreement between the wing crack locations and the particles 473 predominantly loaded in tension. First, before fracturing initiates, a lens of 


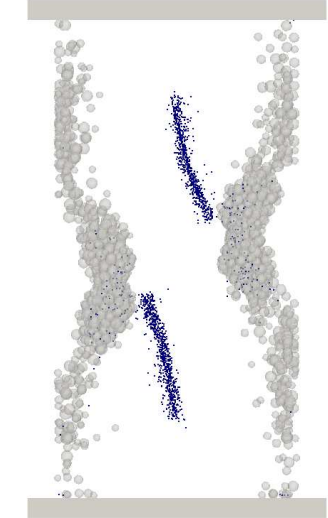

(a) $\sigma \approx 14 \mathrm{MPa}$ $\left(\max \left(\left\|\boldsymbol{s}^{p}\right\|\right) \approx 64 \mathrm{MPa}\right)$

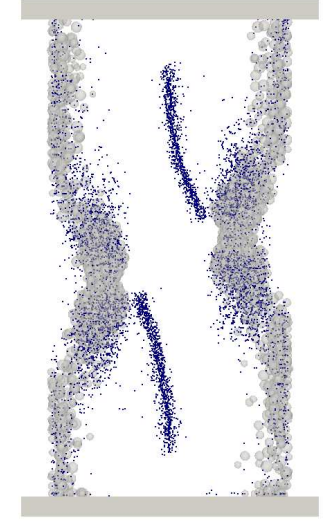

(b) $\sigma \approx 22 \mathrm{MPa}$ $\left(\max \left(\left\|s^{p}\right\|\right) \approx 100 \mathrm{MPa}\right)$

Figure 19: Crack patterns and particles with $\left\|\boldsymbol{s}^{p}\right\|>0.35 \max \left(\left\|\boldsymbol{s}^{p}\right\|\right)$ for a sample with an open flaw $\left(\theta=30^{\circ}\right.$, UCS $\left.=31 \mathrm{MPa}\right)$ under compression. The external plattens are visible to show the extent of the sample.

tensily loaded particles exists around the flaw. Then this lens deforms into a wing pattern along which microcracking occurs. Some stress relaxation is captured after the cracks form: some particles at mid-length of the wing cracks do not obey $\sigma_{I I I}^{p}+\sigma_{I}^{p}<0$ anymore and hence are no longer displayed (Fig. 20 (c) and 20(d)). Finally, it is important to note that secondary cracks are definitely not associated with tensile-loaded particles.

In case of high inclination $\left(\theta \geqslant 60^{\circ}\right)$, the localized damage initiation evocated in Section 4.2 is also associated with tensile stresses. However, tensile loaded zones, i.e., wing cracks do not propagate due to the proximity of highly deviatoric loaded zones where secondary cracks arise afterwards (Fig. 21).

In addition, the rotation of the local major principal stress directions due to the presence of the flaw is captured in Fig. 18(c). On one hand, wing cracks propagate conforming to the deflected local major principal stress directions confirming here their mode I opening nature. On the other hand, secondary cracks occur in areas with local major principal directions oriented almost vertically and seem to propagate along no defined direction. 


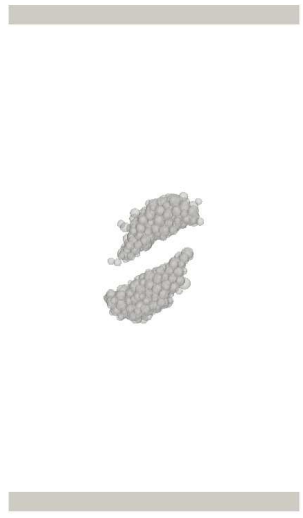

(a) $\sigma \approx 2.5 \mathrm{MPa}$

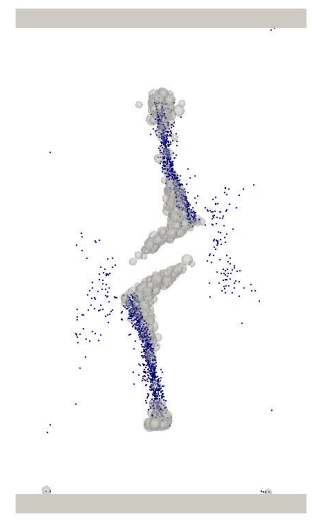

(c) $\sigma \approx 14 \mathrm{MPa}$
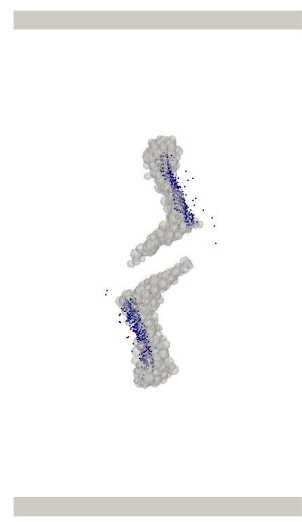

(b) $\sigma \approx 9.5 \mathrm{MPa}$

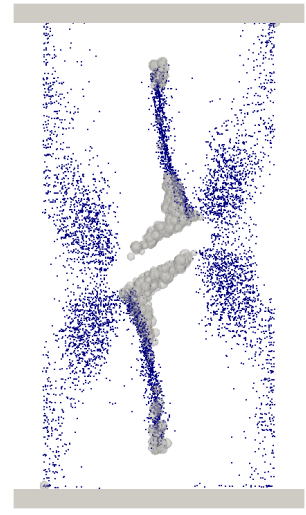

(d) $\sigma \approx 22 \mathrm{MPa}$

Figure 20: Crack patterns and particles with $\sigma_{I I I}^{p}+\sigma_{I}^{p}<0$ for a sample with an open flaw $\left(\theta=30^{\circ}, \mathrm{UCS}=31 \mathrm{MPa}\right)$ under compression. The external plattens are visible to show the extent of the sample. 


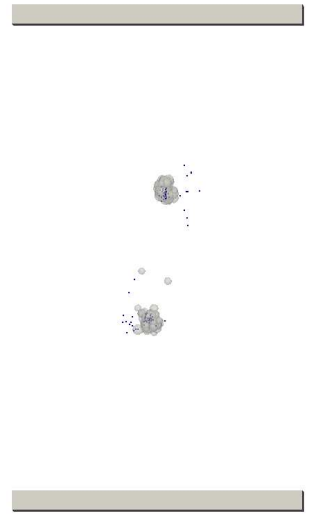

(a) $\sigma \approx 14 \mathrm{MPa}$

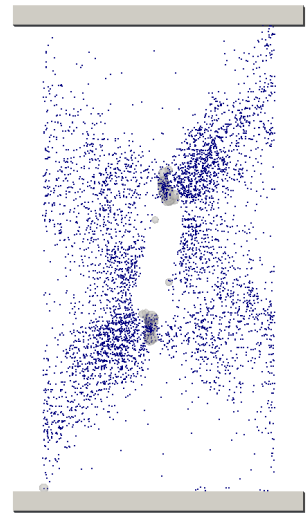

(b) $\sigma \approx 28 \mathrm{MPa}$

Figure 21: Crack patterns and particles with $\sigma_{I I I}^{p}+\sigma_{I}^{p}<0$ for a sample with an open flaw $\left(\theta=75^{\circ}, \mathrm{UCS}=48 \mathrm{MPa}\right)$. The external plattens are visible to show the extent of the sample.

\subsection{Micro-mechanics of closed flaw propagation}

In section 5 , it has been shown that from closed flaw, crack propagation localizes in wing crack for more specific conditions than from open flaws. When wing cracks occur, a very good correlation can again be found between the wing crack locations and the particles sustaining a mainly tensile state of stress $\left(\sigma_{I I I}^{p}+\sigma_{I}^{p}<0\right)$ as illustrated in Fig. 22. For such cases, with $\theta>\phi$, because sliding occurs along the flaw, tensile stresses develop in the sample above each flaw tip. Again, during wing crack extension, tensile stresses develop at the tip of the wing cracks whereas stress relaxation occurs along the wing crack branch (Fig. 22(c)). Once the wing cracks have reached the sample ends, secondary cracks develop due to the concentration of compressive stresses (Fig. 23).

For increasing $\phi$ values, wing crack propagation is inhibited because sliding along the flaw is reduced, limiting the development of tensile stresses at the tip of the flaw. Indeed, particles sustaining tensile stresses (with $\sigma_{I I I}^{p}+\sigma_{I}^{p}<0$ ) are much fewer and are closer to the flaw with $\phi=30^{\circ}$ than with $\phi=0^{\circ}$, for $\theta=45^{\circ}$ (Fig. 24). Ultimately, when $\phi>\theta$, tensile stresses no longer develop in 


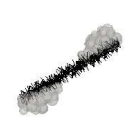

(a) $\sigma \approx 3.0 \mathrm{MPa}$

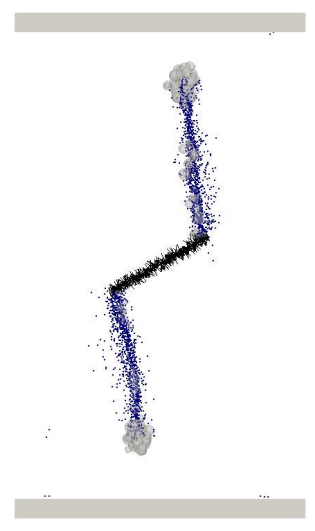

(c) $\sigma \approx 12 \mathrm{MPa}$

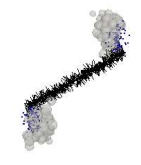

(b) $\sigma \approx 8.3 \mathrm{MPa}$

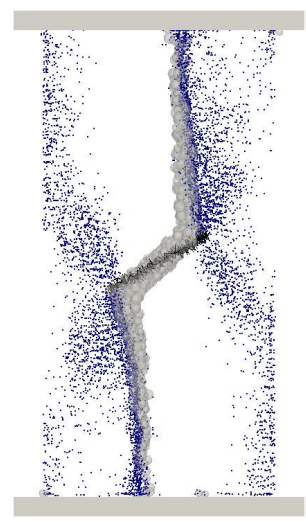

(d) $\sigma \approx 22 \mathrm{MPa}$

Figure 22: Crack patterns and particles with $\sigma_{I I I}^{p}+\sigma_{I}^{p}<0$ for a sample with a frictionless closed flaw $\left(\theta=30^{\circ}\right.$, UCS $\left.=41 \mathrm{MPa}\right)$ under compression. 


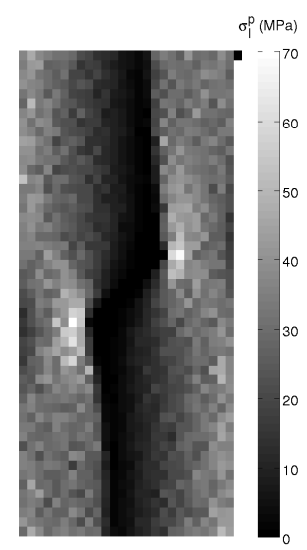

Figure 23: Micro-mechanical field of major principal stress $\sigma_{I}^{p}$ for a closed flaw with $(\theta=$ $\left.30^{\circ} ; \phi=0^{\circ}\right)$ at $\sigma \approx 22 \mathrm{MPa}$. The corresponding crack pattern appears in Fig. 22(d)

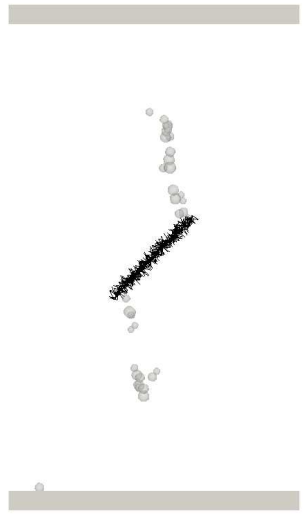

(a) $\phi=0^{\circ}$

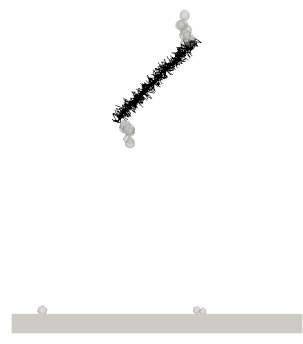

(b) $\phi=30^{\circ}$

Figure 24: Particles with $\sigma_{I I I}^{p}+\sigma_{I}^{p}<0$ for a closed flaw inclined at $\theta=45^{\circ}$ with different friction angles. Under $\varepsilon_{z} 0.04 \% ; \sigma_{z} \in[19 ; 20] \mathrm{MPa}$ : see the Fig. 16(a) for corresponding crack patterns. 
the sample and wing cracks cannot grow. In such cases only a negligible amount of particles with $\sigma_{I I I}^{p}+\sigma_{I}^{p}<0$ is detected.

Despite the tensile local failure mode of all bond breakages in the model, these micro-mechanical insights support the idea of different damage mechanisms causing either primary (wing) or secondary (shear) cracks. This emphasizes the advantage of intermediate- (or meso-) scale mechanical measurements, such as the particle stress tensors, for a better characterization of the stress distribution inside the material.

\section{Conclusion}

Discrete element modeling of crack propagation in rock under uniaxial compression has been discussed. The advantages of the model were presented as well as its limitations. It has been shown that, due to the brittle nature of the local interaction laws used, damping and discrete element size influence quantitatively the simulated strength, but not the elastic properties.

The model enables to simulate explicitly both open and closed flaws with various associated properties. A special attention has been paid to use an adequate discrete model for simulating closed flaws. We showed that a realistic elastic-plastic behavior with controlled properties can be obtained using a SJM formulation with as little numerical bias as possible.

Strengths and crack propagation patterns were obtained for open and closed flaws with different inclinations and favorably compared with existing experimental results. The DEM model offers micro-mechanical insights into the different damage mechanisms occurring in brittle materials. In particular, fracturing localizes in the form of wing cracks at locations where tensile stresses develop. Such wing cracks propagate according to the most compressive stress direction. Diffuse zones of secondary cracks are shown to correspond to excess deviatoric compressive stresses. The distribution of deviatoric compressive and tensile local stresses depend on the nature of the flaw (open or closed) as well as on the frictional strength of the flaw (if closed). 
As such, the flaw friction angle affects crack propagation patterns, as well as the overall strength of the material. Ultimately, when friction increases, wing crack are inhibited and a more diffuse fracturing pattern is observed. That is, crack propagation in the intact material (the matrix) is affected by the flaw properties, and not only by those of the matrix.

The influence of the flaw properties on crack propagation and on the overall behavior emphasizes the need to use the SJM for DEM simulations of closed flaws, unless discrete particles correspond to physical entities. Such a model could then be applied to study crack coalescence under confined states. It also suggests further improvements of the DEM, by applying SJM to newly created cracks. This would be useful for bonds broken in shear mode, for which contacts still hold after bond breakage.

\section{APPENDIX A: representations of $\sigma^{p}$ field}

Micro-mechanical fields such as the one depicted in Fig. 6(b) are illustrated using the following procedure. Because of the reduced thickness of the sample along the $y$ direction $\left(L_{y} \approx L_{x} / 2 \approx L_{z} / 4\right)$, plane stresses in the $(x, z)$ plane are considered.

For a random selection of particles, principal directions of $\sigma^{p}$ can readily be plotted at the location $x, z$ of particles centers (plane projection of the 3D model).

A direct illustration of principal stress values for all $\sigma^{p}(x, y, z)$ being impossible, plane representation of another related field is made as follows. The $(x, z)$ plane is discretized in a square grid with a cell length $d x=d z=0.2$ $\mathrm{m}$, which is around the mean diameter of particles. All particles whose center lies in one given cell $x_{i}, z_{i}$ are identified. Then, Fig. 6, 18 and 23 rely on a micro-mechanical value of the stress inside each cell, denoted $\boldsymbol{\sigma}^{\boldsymbol{p}}\left(x_{i}, z_{i}\right)$, that is derived by summing all internal moment tensors of these particles:

$$
\boldsymbol{\sigma}^{p}\left(x_{i}, z_{i}\right)=\frac{\sum V^{p} \boldsymbol{\sigma}^{p}}{d x d z L_{y}}
$$


If $d x$ and $d z$ are such that one unique cell would include the whole model, equation (A.1) corresponds to the Love-Weber formula and the macroscopic stress, at the REV scale, is obtained.

\section{APPENDIX B: elastic-plastic behavior of a rock joint}

Let us consider an elastic-plastic rock joint. Equation (B.1) rules the elastic behavior:

$$
\left(\begin{array}{c}
d \tau \\
d \sigma
\end{array}\right)=\left(\begin{array}{cc}
K_{t}^{g l o b} & 0 \\
0 & K_{n}^{g l o b}
\end{array}\right)\left(\begin{array}{l}
d \gamma^{e} \\
d u^{e}
\end{array}\right)
$$

The (perfectly) plastic behavior occurs on the yield surface $\tau=\sigma \tan (\phi)$, and plastic deformation depends on the dilatancy angle $\psi$ through the following flow rule: $d u^{p} /\left|d \gamma^{p}\right|=-\tan (\psi)[55]$.

Being loaded under constant normal displacement shear mode $(d u=0)$, under an initial value of normal stress $\sigma \neq 0$, the stress state reaches the plastic limit condition after some elastic deformation. If loading continues, any change in tangential relative displacement is fully plastic from this time: $d \gamma=d \gamma^{p}$. Thus $d u^{p}=-\left|d \gamma^{p}\right| \tan (\psi)=-|d \gamma| \tan (\psi)$. The total normal relative displacement $u$ being constant due to the imposed CND loading, the plastic dilatant behavior of the joint induces changes in the normal stress $\sigma$, as derived in equation (B.2):

$$
d u=d u^{e}+d u^{p}=0 \Leftrightarrow d \sigma / K_{n}^{g l o b}-|d \gamma| \tan (\psi)=0 \Leftrightarrow d \sigma /|d \gamma|=K_{n}^{g l o b} \tan (\psi)
$$

This equation is plotted in Fig. $8(\mathrm{~d})$. For the Mohr plane $(\sigma, \tau)$, the MohrCoulomb plastic limit condition imposes $d \tau=d \sigma \tan (\phi)$, see Fig. 8(c). By combining this last equation with equation (B.2) the theoretical line for the plastic regime in $(\gamma, \tau)$ plane can be deduced, see Fig. $8(\mathrm{~b})$.

\section{Acknowledgments}

The authors thank ANR GeoSMEC (2012-BS06-0016-03) and its principal investigator, Y. Klinger, for funding and stimulating project. 


\section{References}

[1] W. F. Brace, E. G. Bombolakis, A note on brittle crack growth in compression, Journal of Geophysical Research 68 (12) (1963) 3709-3713. doi:10.1029/JZ068i012p03709.

[2] E. Lajtai, Brittle fracture in compression, International Journal of Fracture 10 (4) (1974) 525-536. doi:10.1007/BF00155255.

[3] S. Nemat-Nasser, H. Horii, Compression-induced nonplanar crack extension with application to splitting, exfoliation, and rockburst, Journal of Geophysical Research: Solid Earth 87 (B8) (1982) 6805-6821. doi:10.1029/JB087iB08p06805.

[4] C. Park, A. Bobet, Crack coalescence in specimens with open and closed flaws: A comparison, International Journal of Rock Mechanics and Mining Sciences 46 (5) (2009) 819 - 829. doi:10.1016/j.ijrmms.2009.02.006.

[5] H. Jiefan, C. Ganglin, Z. Yonghong, W. Ren, An experimental study of the strain field development prior to failure of a marble plate under compression, Tectonophysics 175 (13) (1990) 269 - 284, earthquake Source Processes. doi:10.1016/0040-1951(90)90142-U.

[6] A. Bobet, H. Einstein, Fracture coalescence in rock-type materials under uniaxial and biaxial compression, International Journal of Rock Mechanics and Mining Sciences 35 (7) (1998) 863 - 888. doi:10.1016/S01489062(98)00005-9.

[7] N. Cho, C. Martin, D. Sego, Development of a shear zone in brittle rock subjected to direct shear, International Journal of Rock Mechanics and Mining Sciences 45 (8) (2008) 1335 - 1346. doi:10.1016/j.ijrmms.2008.01.019.

[8] L. Wong, H. Einstein, Systematic evaluation of cracking behavior in specimens containing single flaws under uniaxial compression, International Journal of Rock Mechanics and Mining Sciences 46 (2) (2009) 239 - 249. doi:10.1016/j.ijrmms.2008.03.006. 
[9] B. Shen, O. Stephansson, H. H. Einstein, B. Ghahreman, Coalescence of fractures under shear stresses in experiments, Journal of Geophysical Research: Solid Earth 100 (B4) (1995) 5975-5990. doi:10.1029/95JB00040.

[10] N. Cannon, E. Schulson, T. Smith, H. Frost, Wing cracks and brittle compressive fracture, Acta Metallurgica et Materialia 38 (10) (1990) 1955 1962. doi:10.1016/0956-7151(90)90307-3.

[11] L. Germanovich, R. Salganik, A. Dyskin, K. Lee, Mechanisms of brittle fracture of rock with pre-existing cracks in compression, pure and applied geophysics 143 (1-3) (1994) 117-149. doi:10.1007/BF00874326.

[12] H. Lee, S. Jeon, An experimental and numerical study of fracture coalescence in pre-cracked specimens under uniaxial compression, International Journal of Solids and Structures 48 (6) (2011) 979 - 999. doi:10.1016/j.ijsolstr.2010.12.001.

[13] S. P. Morgan, C. A. Johnson, H. H. Einstein, Cracking processes in barre granite: fracture process zones and crack coalescence, International Journal of Fracture 180 (2) (2013) 177-204. doi:10.1007/s10704-013-9810-y.

[14] R. Goldstein, R. Salganik, Brittle fracture of solids with arbitrary cracks, International Journal of Fracture 10 (4) (1974) 507-523. doi:10.1007/BF00155254.

[15] B. Lauterbach, D. Gross, Crack growth in brittle solids under compression, Mechanics of Materials 29 (2) (1998) 81 - 92. doi:10.1016/S01676636(97)00069-0.

[16] J.-B. Leblond, J. Frelat, Crack kinking from an initially closed crack, International Journal of Solids and Structures 37 (11) (2000) 1595 - 1614. doi:10.1016/S0020-7683(98)00334-5.

[17] B. Prabel, A. Combescure, A. Gravouil, S. Marie, Level set x-fem nonmatching meshes: application to dynamic crack propagation in elasticplas- 
tic media, International Journal for Numerical Methods in Engineering 69 (8) (2007) 1553-1569. doi:10.1002/nme.1819.

[18] N. Moës, J. Dolbow, T. Belytschko, A finite element method for crack growth without remeshing, International Journal for Numerical Methods in Engineering 46 (1) (1999) 131-150. doi:10.1002/(SICI)10970207(19990910)46:1;131::AID-NME726¿3.0.CO;2-J.

[19] P. Cundall, O. Strack, A discrete numerical model for granular assemblies, Géotechnique 29 (1979) 47-65.

[20] F. V. Donzé, S. A. Magnier, Formulation of a three-dimensional numerical model of brittle behaviour, Geophys. J. Int. 122 (1995) 790-802.

[21] M. Jirásek, Z. Bazant, Particle model for quasibrittle fracture and application to sea ice, Journal of Engineering Mechanics 121 (9) (1995) 1016-1025. doi:10.1061/(ASCE)0733-9399(1995)121:9(1016).

[22] P. Prochzka, Application of discrete element methods to fracture mechanics of rock bursts, Engineering Fracture Mechanics 71 (46) (2004) $601-618$. doi:10.1016/S0013-7944(03)00029-8.

[23] N. M. Azevedo, J. Lemos, J. R. de Almeida, Influence of aggregate deformation and contact behaviour on discrete particle modelling of fracture of concrete, Engineering Fracture Mechanics 75 (6) (2008) 1569 - 1586. doi:10.1016/j.engfracmech.2007.06.008.

[24] D. Jauffrès, X. Liu, C. L. Martin, Tensile strength and toughness of partially sintered ceramics using discrete element simulations, Engineering Fracture Mechanics 103 (2013) 132 - 140. doi:10.1016/j.engfracmech.2012.09.031.

[25] D. Potyondy, P. Cundall, A bonded-particle model for rock, International Journal of Rock Mechanics and Mining Sciences 41 (8) (2004) 1329 - 1364. doi:10.1016/j.ijrmms.2004.09.011. 
[26] J. F. Hazzard, R. P. Young, S. C. Maxwell, Micromechanical modeling of cracking and failure in brittle rocks, Journal of Geophysical Research: Solid Earth 105 (B7) (2000) 16683-16697. doi:10.1029/2000JB900085.

[27] C. Tang, L. Tham, S. Wang, H. Liu, W. Li, A numerical study of the influence of heterogeneity on the strength characterization of rock under uniaxial tension, Mechanics of Materials 39 (4) (2007) 326 - 339. doi:10.1016/j.mechmat.2006.05.006.

[28] Y. Wang, F. Tonon, Calibration of a discrete element model for intact rock up to its peak strength, International Journal for Numerical and Analytical Methods in Geomechanics 34 (5) (2010) 447-469. doi:10.1002/nag.811.

[29] L. Scholtès, F.-V. Donzé, A dem model for soft and hard rocks: Role of grain interlocking on strength, Journal of the Mechanics and Physics of Solids 61 (2) (2013) 352 - 369. doi:10.1016/j.jmps.2012.10.005.

[30] Y. Wang, X. Yin, F. Ke, M. Xia, K. Peng, Numerical simulation of rock failure and earthquake process on mesoscopic scale, Pure and Applied geophysics 157 (11-12) (2000) 1905-1928. doi:10.1007/PL00001067.

[31] X.-P. Zhang, L. N. Y. Wong, Cracking processes in rock-like material containing a single flaw under uniaxial compression: A numerical study based on parallel bonded-particle model approach, Rock Mechanics and Rock Engineering 45 (5) (2012) 711-737. doi:10.1007/s00603-011-0176-z.

[32] Y. Wang, P. Mora, Modeling wing crack extension: Implications for the ingredients of discrete element model, Pure and Applied Geophysics 165 (34) (2008) 609-620. doi:10.1007/s00024-008-0315-y.

[33] L. Scholtès, F.-V. Donzé, Modelling progressive failure in fractured rock masses using a $3 \mathrm{~d}$ discrete element method, International Journal of Rock Mechanics and Mining Sciences 52 (2012) 18 - 30. doi:10.1016/j.ijrmms.2012.02.009. 
[34] V. Šmilauer, E. Catalano, B. Chareyre, S. Dorofeenko, J. Duriez, A. Gladky, J. Kozicki, C. Modenese, L. Scholtès, L. Sibille, J. Stránský, K. Thoeni, Yade Documentation, 1st Edition, The Yade Project, 2010, http: //yade-dem.org.

[35] J. Kozicki, F.-V. Donzé, Applying an open-source software for numerical simulations using finite element or discrete modelling methods, Computer Methods in Applied Mechanics and Engineering 197 (49-50) (2008) 44294443.

[36] F. Donzé, J. Bouchez, S. Magnier, Modeling fractures in rock blasting, International Journal of Rock Mechanics and Mining Sciences 34 (8) (1997) 1153 - 1163. doi:10.1016/S1365-1609(97)80068-8.

[37] H. Huang, B. Lecampion, E. Detournay, Discrete element modeling of tool-rock interaction i: rock cutting, International Journal for Numerical and Analytical Methods in Geomechanics 37 (13) (2013) 1913-1929. doi:10.1002/nag.2113.

[38] N. Cho, C. Martin, D. Sego, A clumped particle model for rock, International Journal of Rock Mechanics and Mining Sciences 44 (7) (2007) 997 1010. doi:10.1016/j.ijrmms.2007.02.002.

[39] X. Ding, L. Zhang, A new contact model to improve the simulated ratio of unconfined compressive strength to tensile strength in bonded particle models, International Journal of Rock Mechanics and Mining Sciences 69 (2014) 111 - 119. doi:10.1016/j.ijrmms.2014.03.008.

[40] D. Potyondy, A flat-jointed bonded-particle material for hard rock, in: 46th US Rock Mechanics/Geomechanics Symposium, ARMA, 2012.

[41] T. Waltham, Foundations of Engineering Geology (Third ed.), Taylor \& Francis, 2009. 
[42] P. A. Cundall, Distinct element models of rock and soil structure, in: E. T. Brown (Ed.), Analytical and Computational Methods in Engineering Rock Mechanics, George Allen and Unwin, 1987, pp. 129-163.

[43] A. Love, A treatise on the mathematical theory of elasticity, Cambridge University Press, Cambridge, 1927.

[44] J. Weber, Recherches concernant les contraintes intergranulaires dans les milieux pulvérulents, Bulletin de liaison des Ponts et Chaussées 20 (1966) $1-20$.

[45] W. L. Lim, G. R. McDowell, Discrete element modelling of railway ballast, Granular Matter 7 (1) (2005) 19-29. doi:10.1007/s10035-004-0189-3.

[46] A. A. Griffith, The phenomena of rupture and flow in solids, Philosophical Transactions of the Royal Society of London. Series A, Containing Papers of a Mathematical or Physical Character 221 (1920) 163-198.

[47] J. Lemaitre, J.-L. Chaboche, Mechanics of solid materials, Cambridge university press, 1990.

[48] J.-J. Moreau, Numerical investigation of shear zones in granular materials, in: D. E. Wolf, P. Grassberger (Eds.), Friction Arching, Contact Dynamics, World Scientific, Balkema, 1997, pp. 233-247.

[49] L. Staron, F. Radjai, J.-P. Vilotte, Multi-scale analysis of the stress state in a granular slope in transition to failure, The European Physical Journal E 18 (3) (2005) 311-320. doi:10.1140/epje/e2005-00031-0.

[50] D. M. Ivars, M. E. Pierce, C. Darcel, J. Reyes-Montes, D. O. Potyondy, R. P. Young, P. A. Cundall, The synthetic rock mass approach for jointed rock mass modelling, International Journal of Rock Mechanics and Mining Sciences 48 (2) (2011) 219 - 244. doi:10.1016/j.ijrmms.2010.11.014.

[51] M. Bahaaddini, G. Sharrock, B. Hebblewhite, Numerical investigation of the effect of joint geometrical parameters on the mechanical properties of 
a non-persistent jointed rock mass under uniaxial compression, Computers and Geotechnics 49 (2013) 206 - 225. doi:10.1016/j.compgeo.2012.10.012.

[52] P. Kulatilake, B. Malama, J. Wang, Physical and particle flow modeling of jointed rock block behavior under uniaxial loading, International Journal of Rock Mechanics and Mining Sciences 38 (5) (2001) 641 - 657. doi:10.1016/S1365-1609(01)00025-9.

[53] F. Radjai, S. Roux, J. J. Moreau, Contact forces in a granular packing, Chaos: An Interdisciplinary Journal of Nonlinear Science 9 (3) (1999) 544550. doi:10.1063/1.166428.

[54] H. Li, L. N. Y. Wong, Influence of flaw inclination angle and loading condition on crack initiation and propagation, International Journal of Solids and Structures 49 (18) (2012) 2482 - 2499. doi:10.1016/j.jjsolstr.2012.05.012.

[55] J. Duriez, F. Darve, F.-V. Donzé, Incrementally non-linear plasticity applied to rock joint modelling, International Journal for numerical and analytical methods in geomechanics 37 (5) (2013) 453-477. doi:10.1002/nag.1105. 